\title{
Detecting Photoacoustic Signals of Sulfur Hexafluoride at Varying Microphone Positions
}

\author{
Wittmann S. Murphy, Han Jung Park \\ Department of Chemistry and Physics, University of Tennessee at Chattanooga, Chattanooga, TN, USA \\ Email: hanjung-park@utc.edu
}

Received 28 April 2016; accepted 24 June 2016; published 27 June 2016

Copyright (C) 2016 by authors and Scientific Research Publishing Inc.

This work is licensed under the Creative Commons Attribution International License (CC BY).

http://creativecommons.org/licenses/by/4.0/

(c) (i) Open Access

\begin{abstract}
Photoacoustic spectroscopy was used to test the photoacoustic properties of sulfur hexafluoride, an optically thick and potent greenhouse gas. While exploring the photoacoustic effect of sulfur hexafluoride, the effects of the position of the microphone within a gas cell were determined. Using a $35 \mathrm{~cm}$ gas cell, microphones were positioned at $17.5 \mathrm{~cm}$, the middle of the gas cell, $12.5 \mathrm{~cm}$, $7.5 \mathrm{~cm}$, and $2.5 \mathrm{~cm}$ from the window of the cell. From the photoacoustic signal produced for each resonance frequency at each microphone position, the effects of acoustic pressure produced at each position on the signal recorded were observed. This is the first study done by experimentation with the photoacoustic effect to show that standing waves have different amplitudes at different microphone positions.
\end{abstract}

\section{Keywords}

Photoacoustic Effect, Sulfur Hexafluoride, Gas Detection, Microphone Placement, Acoustic Wave Formation

\section{Introduction}

In 1880, Alexander Graham Bell discovered the phenomenon that was the photoacoustic effect [1]. Through experimentations with his photophone, Bell's discoveries determined that measureable acoustic waves could be detected when modulated optical radiation was directed towards a gaseous, liquid, or solid material [2]. The light directed onto an object results in thermal excitation of the object, thus resulting in thermal expansion. However, since the light is modulated, excitation as well as de-excitation takes place causing expansion and contraction of the molecules making up the material. The periodic expansion and contraction of the material's 
molecules result in the production of detectable and measurable acoustic waves [3].

Due to the acoustic waves produced by the photoacoustic effect, pressure builds up within a gas cell. At resonance frequencies, the acoustic pressure created forms areas of maximum amplitudes within the cell. A microphone positioned within the cell is capable of detecting the audible pressure fluctuations, and an oscilloscope is used to convert the detected signals into a visible waveform for recording [4]. A diagram displaying the pressure amplitudes within the gas cell at different resonance frequencies can be seen in Figure 1 [5]. The signal amplitude, displayed by the oscilloscope, is capable of reflecting the amplitude of acoustic pressure at that particular location and resonance [6]. Through the placement of microphones at varying positions across a gas cell, the photoacoustic signal generated can be detected and compared to the pressure fluctuations and their amplitudes at these varying positions as represented by Figure 1. Sulfur hexafluoride $\left(\mathrm{SF}_{6}\right)$ is a man-made gas used in electrical transmission equipment, semiconductor manufacturing, and has become the most commonly used insulating gas in electrical systems. Due to the increased production of $\mathrm{SF}_{6}$ and its prolonged lifetime within the atmosphere, detection of its leaks has become a growing concern. Since $\mathrm{SF}_{6}$ is a strongly absorbing gas, with a large optical absorption coefficient at a wavelength of $10.6 \mu \mathrm{m}$, it is the ideal gas for detection using photoacoustic spectroscopy. The large absorbance peak at $10.6 \mu \mathrm{m}$ is the reason for using a $\mathrm{CO}_{2}$ laser here [7]. In this research, the effects of the position of the microphone within a gas cell containing $\mathrm{SF}_{6}$ were determined.

\section{Methods/Procedures}

A $35 \mathrm{~cm}$ gas cell was constructed using three $10 \mathrm{~cm}$ gas cells and one $5 \mathrm{~cm}$ gas cell (RJ Spectroscopy Company). Four electret microphone elements (RadioShack, 270-092) were fixed to the top of four inlet/outlet ports using short segments of rubber tubing and Parafilm laboratory film. The microphones were placed so that one microphone was positioned in the middle of the gas cell, $17.5 \mathrm{~cm}$, and the other microphones were positioned at 12.5 , 7.5 , and $2.5 \mathrm{~cm}$ from one of the gas cell's germanium windows (Thorlabs, Inc.). The germanium windows were used due to their low absorption at the wavelength of the laser used and to minimize the effects of moisture on the experiment. The microphones were connected to a voltage amplifier (Femto, Model HVA-10M-60-B) and a digital oscilloscope (Tektronix, TBS 1202B). A diagram of the gas cell and the placement of the microphones can be found in Figure 2.

The microphone positioned at $17.5 \mathrm{~cm}$ was connected to a power source and the oscilloscope. After filling the gas cell with $\mathrm{SF}_{6}$, a $\mathrm{CO}_{2}$ laser (Access Laser Company, L3) was used to excite the gas molecules. Since the laser used is a continuous laser, an optical chopper (Thorlabs, Inc.) was placed in the middle of the alignment of the $\mathrm{CO}_{2}$ laser and the gas cell in order to modulate the light. Also, a $600-700 \mathrm{~nm}$ laser (Thorlabs, Inc., CPS182) was used as a reference laser and detected by a Si photo detector (Thorlabs, Inc., DET36A). The Si detector was also connected to the oscilloscope. A diagram of the experimental setup used can be seen in Figure 3. The

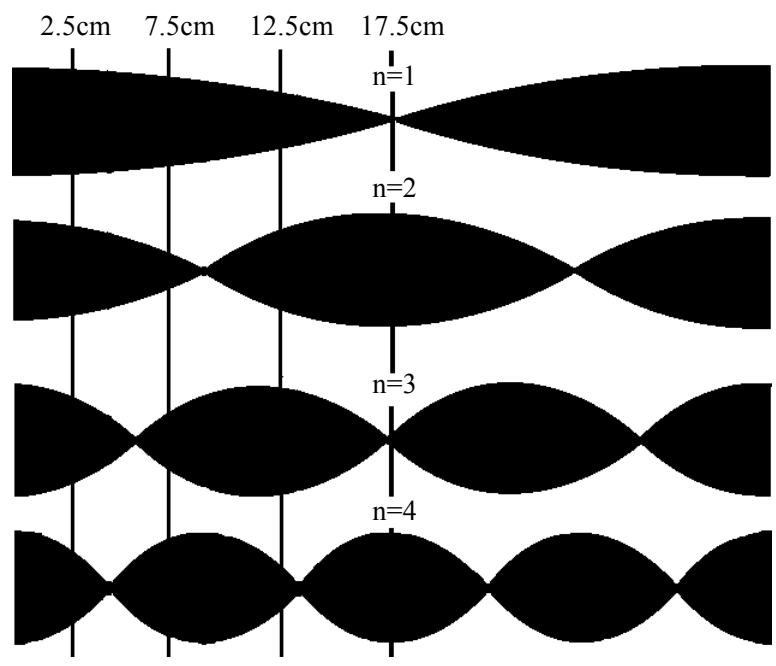

Figure 1. Diagram of pressure waves formed at the first four resonance frequencies with microphone placement [5]. 


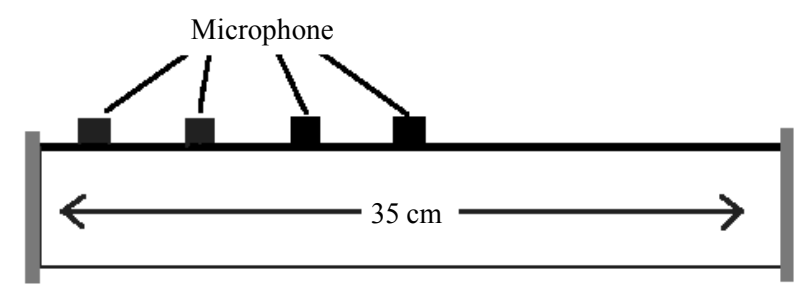

Figure 2. Diagram of gas cell setup with microphones.

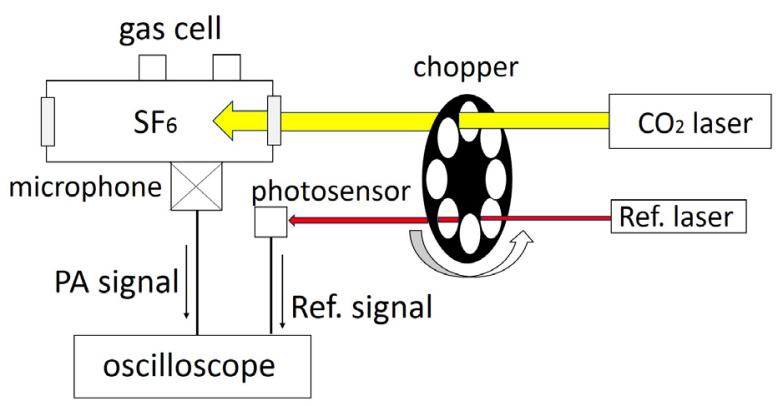

Figure 3. Diagram of experimental setup.

amplitude of the sound waves produced was measured by the oscilloscope and recorded for each frequency increment using $10 \mathrm{~Hz}$ increments from $100 \mathrm{~Hz}$ to $1000 \mathrm{~Hz}$. When a resonance frequency was found, $1 \mathrm{~Hz}$ increments, the lowest possible increment for the chopper used, were used to determine the exact frequency. Each resonance frequency was measured to its precise frequency and recorded. This process was also completed for the microphones positioned at $12.5,7.5$, and $2.5 \mathrm{~cm}$.

In order to show reproducibility, the procedure and process previously mentioned was completed for a total of three times over a period of two weeks. Similar experimental conditions were provided for each trial: room temperature, one-third power setting for $\mathrm{CO}_{2}$ laser, and a $35 \mathrm{~cm}$ gas cell containing $100 \% \mathrm{SF}_{6}$. Data in Figures 4-7 represent the average of 64 replicates.

\section{Results and Discussion}

Within the gas cell, nodes are formed at each resonance frequency due to the amplitude of pressure caused by the acoustic waves [5]. As previously mentioned, a representation of the pressure waves produced due to the photoacoustic effect can be found in Figure 1. Resonance frequencies were determined for each microphone position. The recorded measurement of the photoacoustic signal determined at each of these resonance frequencies can be found in Table 1. The signal versus frequency plot for positions $2.5,7.5,12.5,17.5 \mathrm{~cm}$ can be found as Figures 4-7, respectively.

Since a $35 \mathrm{~cm}$ gas cell was used, the microphone positioned at $17.5 \mathrm{~cm}$ is in the middle of the cell, and the microphone positioned at $2.5 \mathrm{~cm}$ is most towards the outer edge of the diagram found in Figure 1. The amplitude of the pressure wave at the position of the microphone at each resonance should correspond to the signal detected by the microphone at that resonance. The results obtained from Figures 4-7 confirm the previous assumption. At low-pressure wave amplitudes the signal is lower in strength than at high-pressure wave amplitudes. The results obtained were consistent for all four of the positions.

Due to the optical thickness and high absorption coefficient of $\mathrm{SF}_{6}$ at a wavelength of $10.6 \mu \mathrm{m}$ most of the light from the $\mathrm{CO}_{2}$ laser is absorbed at the incident point at which the light comes into contact with the gas [8]. Because of the high absorbance of light at this part of the gas cell, the corresponding photoacoustic signal produced should be the maximum signal recorded at the first resonance frequency. From the data collected in Table 1 , the previous statement stands true.

\section{Conclusion}

The effects of the position of a microphone within a gas cell containing $\mathrm{SF}_{6}$, an optically thick, greenhouse gas, 


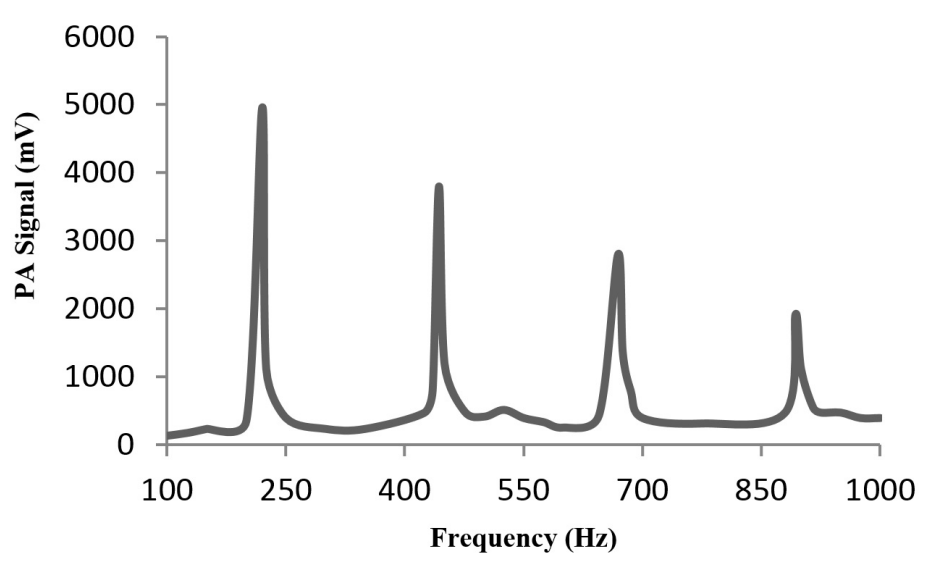

Figure 4. Photoacoustic signal vs. frequency for microphone positioned at $2.5 \mathrm{~cm}$.

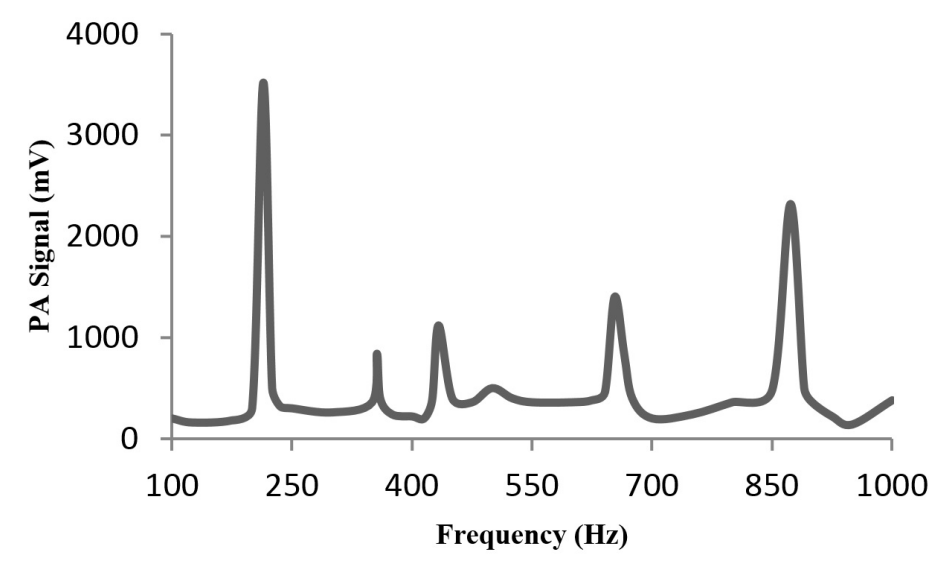

Figure 5. Photoacoustic signal vs. frequency for microphone positioned at $7.5 \mathrm{~cm}$.

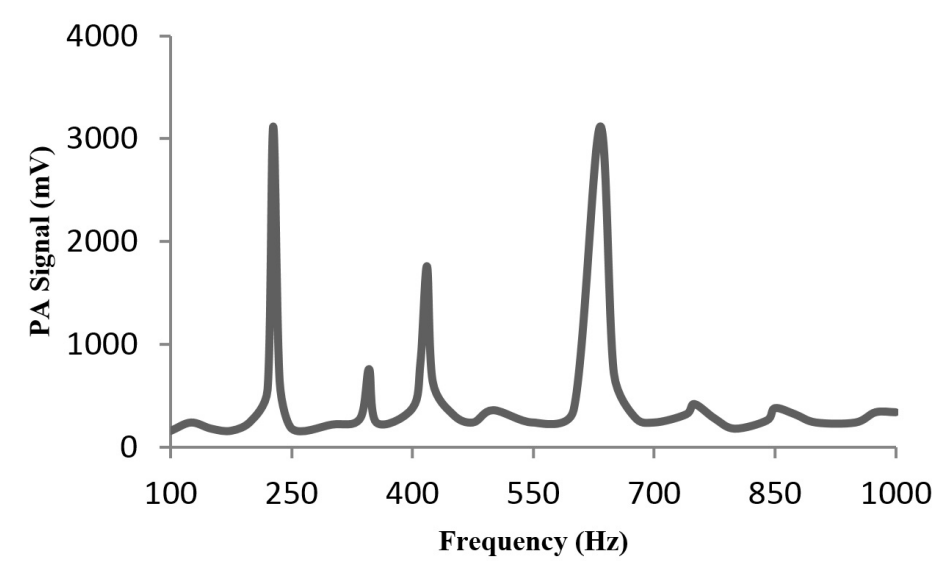

Figure 6. Photoacoustic signal vs. frequency for microphone positioned at $12.5 \mathrm{~cm}$.

have been determined. Through the use of a photoacoustic spectroscopy technique and detection of pressure amplitudes within the gas cell, it was determined that the signals detected from the microphones corresponded to the amplitude of pressure waves at particular resonance frequencies within particular areas of the gas cell. The position of a microphone within a gas cell is an experimental condition that has yet to be tested to determine its 


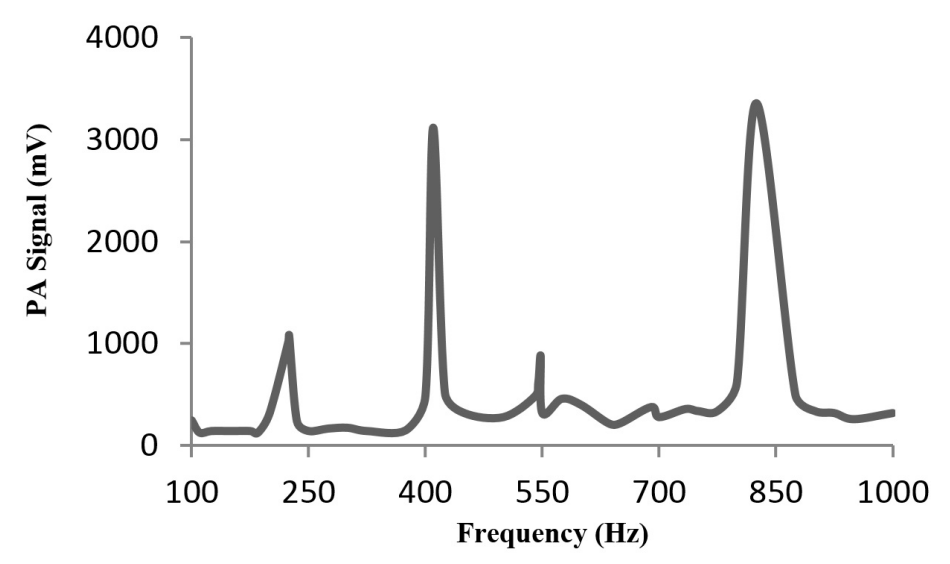

Figure 7. Photoacoustic signal vs. frequency for microphone positioned at $17.5 \mathrm{~cm}$.

Table 1. PA signal detected at each resonance frequency for varying microphone positions.

\begin{tabular}{ccccc}
\hline $\begin{array}{c}\text { Position of Microphone } \\
(\mathrm{cm})\end{array}$ & $\begin{array}{c}\text { Signal at 1st Resonance } \\
(\mathrm{mV})\end{array}$ & $\begin{array}{c}\text { Signal at 2nd Resonance } \\
(\mathrm{mV})\end{array}$ & $\begin{array}{c}\text { Signal at 3rd Resonance } \\
(\mathrm{mV})\end{array}$ & $\begin{array}{c}\text { Signal at 4th Resonance } \\
(\mathrm{mV})\end{array}$ \\
\hline 2.5 & 4960 & 3800 & 2800 & 1920 \\
7.5 & 3520 & 1120 & 1400 & 2320 \\
12.5 & 3120 & 1760 & 3120 & 380 \\
17.5 & 1080 & 3120 & 380 & 3360 \\
\hline
\end{tabular}

effect on detecting photoacoustic pressure signals. The data collected can be beneficial in the positioning of microphones for detecting trace amounts of gas if the " $n$ " value of the resonance frequency is known. The results of this experimentation display how resonance frequency, peak amplitude useful in trace amount detection, is related to the proper position of the microphone, a technique that can be advantageous in conventional photoacoustic experiments. This experiment is also the first study to use the photoacoustic effect to show that standing waves have different amplitudes at different positions.

\section{Acknowledgements}

The authors would like to thank the University of Tennessee at Chattanooga Grote Chemistry fund and the Provost Start-up Grants for their funding and support of the research conducted.

\section{References}

[1] Na Hecke, G.R. and Karukstis, K.K. (1998) Photoacoustic Thermal Characterization of Liquid Crystals. In: Hyde, C.W., Ed., A Guide to Lasers in Chemistry, Jones and Bartlett Publishers, Inc., Massachusetts, 169-177.

[2] Lüscher, E., Coufal, H.J., Korpiun, P. and Tilgner, R. (1984) Photoacoustic Detection and Monitoring. In: Photoacoustic Effect Principles and Applications: Proceedings of the First International Conference on the Photoacoustic Effect in Germany, Vieweg+Teubner Verlag, Braunschweig, 188-189.

[3] Miklós, A., Schäfer, S. and Hess, P. (2010) Photoacoustic Spectroscopy, Theory. In: Lindon, J.C., Ed., Encyclopedia of Spectroscopy and Spectrometry, 2nd Edition, Academic Press, New York, 1815-1822.

[4] Chen, M. (2011) Method for Detecting Sulfur Dioxide Concentration via Photoacoustic Spectrometry. CN102226752A.

[5] Yehya, F. and Chaudhary, A.K. (2011) Designing and Modeling of Efficient Resonant Photo Acoustic Sensors for Spectroscopic Applications. Journal of Modern Physics, 2, 200-209. http://dx.doi.org/10.4236/jmp.2011.24028

[6] Besson, J.-P., Schilt, S. and Thevenaz, L. (2006) Sub-ppm Multi-Gas Photoacoustic Sensor. Spectrochimica Acta Part $A, \mathbf{6 3}, 899-904$. http://dx.doi.org/10.1016/i.saa.2005.10.034

[7] Sulfur Hexafluoride (2009) NIST Standard Reference Data Program. 2011 ed.

[8] Al-Hawat, S., Saloum, S. and Zidan, M.D. (2004) Absorption of $\mathrm{CO}_{2}$ Laser Emission by Freon-12. Applied Physics B, 78, 439-442. http://dx.doi.org/10.1007/s00340-003-1376-3 